\title{
A Redução do Colágeno Tipo I está Associada ao Aumento da Atividade da Metaloproteinase-2 e da Expressão Proteica de Leptina no Miocárdio de Ratos Obesos
}

\author{
Decreased Collagen Type I Is Associated With Increased Metalloproteinase-2 Activity And Protein Expression \\ Of Leptin In The Myocardium Of Obese Rats
}

Danielle Cristina Tomaz da Silva-Bertani, ${ }^{1 \oplus}$ Danielle Fernandes Vileigas, ${ }^{1}$ Gustavo Augusto Ferreira Mota, ${ }^{1}$ Sérgio Luiz Borges de Souza, ${ }^{1}$ Loreta Casquel De Tomasi, ${ }^{1}$ Dijon Henrique Salomé de Campos, ${ }^{1 \oplus}$ Adriana Fernandes de Deus, ${ }^{1 \oplus}$ Paula Paccielli Freire, ${ }^{2 \bullet}$ Carlos Augusto Barnabe Alves, ${ }^{2}$ Carlos Roberto Padovani, ${ }^{2}$ Antonio Carlos Cicogna ${ }^{1} \odot$

Faculdade de Medicina de Botucatu - Universidade Estadual Paulista (UNESP), ${ }^{1}$ Botucatu, SP - Brasil

Instituto de Biociências - Universidade Estadual Paulista (UNESP), ${ }^{2}$ Botucatu, SP - Brasil

\section{Resumo}

Fundamento: A obesidade é um fator de risco para complicações médicas, incluindo o sistema cardiovascular. Há informações limitadas sobre o colágeno no coração obeso. Nosso estudo anterior demonstrou uma redução dos níveis proteicos de colágeno miocárdico tipo I em ratos obesos alimentados com uma dieta com alto teor de gordura durante 34 semanas. No entanto, os mecanismos responsáveis pelos níveis baixos não estão completamente elucidados.

Objetivo: O objetivo deste estudo foi testar a hipótese de que a redução do colágeno tipo I está associada ao aumento da atividade da metaloproteinase-2 (MMP-2), a qual está ligada à elevação de leptina no miocárdio de ratos obesos.

Métodos: Ratos Wistar machos com 30 dias de idade foram randomizados em dois grupos: controle (dieta padrão) e obeso (dieta com alto teor de gordura), e alimentados durante 34 semanas. Foram avaliados as características gerais dos animais e os perfis metabólicos e endócrinos. Foram avaliados as expressões proteicas miocárdicas de colágeno tipo I, leptina e inibidores teciduais de metaloproteinases (TIMP), bem como a atividade da MMP-2. O teste de correlação de Pearson foi aplicado para determinar as associações entre variáveis. $\mathrm{O}$ nível de significância foi de $5 \%$.

Resultados: Os animais obesos apresentaram índice de adiposidade mais elevado em comparação ao controle. Foram observadas comorbidades como intolerância à glicose, hiperinsulinemia, resistência à insulina, hiperleptinemia e hipertensão nos ratos obesos. A obesidade reduziu o colágeno tipo I, TIMP-1 e TIMP-2, e aumentou a leptina e a MMP-2 no miocárdio. Houve uma correlação negativa entre o colágeno tipo I e a MMP-2 e uma correlação positiva entre a leptina e a MMP-2.

Conclusão: Foi confirmada a hipótese de que a redução do colágeno tipo I está associada ao aumento da atividade da MMP-2 e da expressão de leptina no miocárdio de ratos obesos. (Arq Bras Cardiol. 2020; 115(1):61-70)

Palavras-chave: Doenças Cardiovasculares/fisiopatologia; Obesidade; Colágeno Tipo 1; Ratos; Leptina; Adiposidade; Inibidores Teciduais de Metaloproteinases; Metaloproteinase-2.

\footnotetext{
Abstract

Background: Obesity is a risk factor for medical complications, including the cardiovascular system. There is limited information on collagen in the heart in obesity. Our previous study showed decreased protein levels of myocardial collagen type I in obese rats fed a high-fat diet for 34 weeks. However, the mechanisms responsible for low levels are not fully elucidated.

Objective: The purpose of this study was to test the hypothesis that the reduction in collagen type I is associated with increased metalloproteinase-2 (MMP-2) activity, which is linked to elevated leptin in the myocardium of obese rats.

Methods: Thirty-day-old male Wistar rats were randomized into two groups, control (standard diet) and obese (high-fat diet), and fed for 34 weeks. The general animal characteristics and metabolic and endocrine profiles were evaluated. Myocardial protein expressions of collagen I, leptin, tissue inhibitors of metalloproteinases (TIMP), and MMP-2 activity were assessed. Pearson correlation was employed to determine the associations between variables. The level of significance was $5 \%$.
}

Correspondência: Antonio Carlos Cicogna •

Faculdade de Medicina de Botucatu, Universidade Estadual de São Paulo (UNESP) - Avenida Prof. Mário Rubens Guimarães Montenegro, s/n. CEP 18618687, Botucatu, SP - Brasil

E-mail: ac.cicogna@unesp.br

Artigo recebido em 13/08/2018, revisado em 27/06/2019, aceito em 17/07/2019

DOI: https://doi.org/10.36660/abc.20180143 
Results: The obese animals had increased adiposity index compared to control. Comorbidities such as glucose intolerance, hyperinsulinemia, insulin resistance, hyperleptinemia, and hypertension were observed in obese rats. Obesity reduced collagen I, TIMP-1, and TIMP-2, and it increased leptin and MMP-2 in the myocardium. There was a negative correlation between collagen I and MMP-2 and a positive correlation between leptin and MMP-2

Conclusion: The hypothesis was confirmed; the reduction in collagen type I is associated with increased MMP-2 activity and leptin expression in the myocardium of obese rats. (Arq Bras Cardiol. 2020; 115(1):61-70)

Keywords: Cardiovascular Diseases/physiopathology; Obesity; Collagen Type 1; Rats; Leptin; Adiposity; Tissue Inhibitor of Metalloproteinases; Metaloproteinase-2

Full texts in English - http://www.arquivosonline.com.br

\section{Introdução}

A obesidade é uma doença metabólica crônica caracterizada pelo acúmulo excessivo de tecido adiposo. A prevalência da obesidade tem aumentado mundialmente, representando um problema grave de saúde pública que afeta tanto países desenvolvidos quanto países em desenvolvimento. ${ }^{1,2}$

Os adipócitos são influenciados por várias substâncias, e secretam numerosos peptídeos que atuam no sistema cardiovascular de maneira direta ou indireta. Portanto, o tecido adiposo não é simplesmente um depósito de energia, mas também um órgão endócrino, parácrino e autócrino ativo com múltiplas funções, incluindo a capacidade de sintetizar e liberar mediadores, como a leptina, que participa de múltiplos processos biológicos, incluindo aqueles que ocorrem no coração. ${ }^{3}$

O coração é composto por miócitos, nervos, vasos e a matriz extracelular (MEC). O componente principal da MEC é o colágeno, predominantemente do tipo I e III, sendo o tipo I o mais abundante, correspondendo a aproximadamente $80 \%$ do colágeno miocárdico total. ${ }^{4}$ Esta proteína é produzida por fibroblastos e degradada pela família das metaloproteinases de matriz (MMP). ${ }^{5}$ Em uma condição estável, o colágeno contribui para a manutenção da arquitetura e função cardíaca. ${ }^{6}$ Vários mecanismos atuam para garantir que os componentes da degradação da matriz por MMP sejam precisamente controlados, incluindo os inibidores teciduais de metaloproteinases (TIMP). ${ }^{7}$ O colágeno cardíaco muda em resposta a estímulos neuro-hormonais e mecânicos, ${ }^{6,8}$ devido a síntese elevada e degradação diminuída ou vice versa.

Vários estudos têm analisado a expressão do colágeno tipo I em diferentes tecidos em modelos experimentais de obesidade. ${ }^{9-11}$ Há informações limitadas sobre o comportamento desse tipo de colágeno no coração de animais obesos. Embora Carroll et al. ${ }^{12}$ tenham demonstrado uma elevação em colágeno miocárdico tipo I em coelhos obesos que receberam uma dieta com alto teor de gordura durante 12 semanas, um estudo prévio do nosso grupo, Silva et al., ${ }^{13}$ encontrou uma redução do colágeno miocárdico tipo I em ratos obesos que receberam uma dieta com alto teor de gorduras insaturadas durante 30 semanas. ${ }^{13}$ No entanto, não foram estudados os mecanismos responsáveis pela redução desse colágeno.

Um dos possíveis mecanismos envolvidos na regulação do colágeno miocárdico tipo I é o aumento do hormônio leptina. ${ }^{5,14-16}$ Apoiando tal hipótese, a maioria dos estudos in vitro tem demonstrado que a leptina aumenta a atividade da MMP-2, ${ }^{5,15,16}$ que está envolvida na degradação do colágeno tipo I. Portanto, o objetivo do presente estudo foi testar a hipótese de que a redução do colágeno miocárdico tipo I, associada com o aumento da atividade da MMP-2, está ligada à elevação de leptina em ratos obesos.

\section{Materiais e métodos}

\section{Animais e protocolo experimental}

Após uma semana de aclimatação, ratos Wistar machos com 30 dias de idade foram aleatoriamente designados, por sorteio, para um dos dois grupos: controle $(n=20)$ e obeso $(\mathrm{n}=21)$. O tamanho da amostra utilizada neste estudo foi baseado na literatura e nos nossos estudos prévios. ${ }^{13,17-19} \mathrm{O}$ grupo controle foi alimentado com ração de ratos padrão (RC Focus 1765, Agroceres $^{\circledR}$, Rio Claro, SP, Brasil) contendo 12,3\% de quilocalorias de gordura, $57,9 \%$ de carboidratos e $29,8 \%$ de proteína, enquanto o grupo obeso foi alimentado com uma das quatro alternantes dietas com alto teor de gordura (RC Focus 2413, 2414, 2415 e 2416, Agroceres $^{\circledR}$, Rio Claro, SP, Brasil) contendo 49,2\% de quilocalorias de gordura, 28,9\% de carboidratos e $21,9 \%$ de proteína. As quatro dietas com alto teor de gordura tinham a mesma composição nutricional, exceto aditivos aromatizantes, a saber, queijo, bacon, chocolate ou baunilha. Cada dieta foi alternada diariamente, e os ratos mantiveram suas respectivas dietas durante 34 semanas consecutivas. A dieta com alto teor de gordura foi caloricamente rica em comparação com a dieta padrão (3,65 kcal/g vs. 2,95 kcal/g) devido à maior composição de gordura. A dieta com alto teor de gordura consistia em ácidos graxos saturados e insaturados, que forneciam $20 \%$ e $80 \%$ das calorias derivadas de gordura, respectivamente.

Os ratos foram alojados em gaiolas individuais em uma sala de ar limpo com controle ambiental a 23 ( \pm 3$)^{\circ} \mathrm{C}$ com um ciclo claro/escuro de 12 horas e umidade relativa de $60 \%$ ( $\pm 5 \%$ ). Todos os experimentos e procedimentos foram realizados de acordo com o Guia para o Cuidado e Uso de Animais de Laboratório, publicado pelo Conselho Nacional de Pesquisa (1996), ${ }^{20}$ e foram aprovados pelo Comitê de Ética da Faculdade de Medicina de Botucatu (UNESP, Botucatu, SP, Brasil, Protocolo: 861-2011).

\section{Características gerais dos animais e perfis metabólicos e endócrinos}

As características gerais dos animais e os perfis metabólicos e endócrinos foram avaliados de acordo com os seguintes parâmetros: peso corporal, gordura corporal (GC), índice de adiposidade (IA), consumo alimentar, ingestão de calorias, 
eficiência alimentar, tolerância à glicose, resistência à insulina, perfil lipídico sérico e concentrações séricas de leptina e insulina. Foi aplicado um critério baseado no IA para determinar a obesidade. O IA é um método fácil e consistente utilizado por vários autores para avaliar a quantidade de GC em roedores. ${ }^{21-23}$

O consumo alimentar e peso corporal foram medidos semanalmente. Foi determinada a ingestão calórica multiplicando o valor energético de cada dieta ( $\mathrm{g} \times \mathrm{kcal}$ ) e consumo alimentar semanal. Para analisar a capacidade dos animais de converter energia alimentar consumida em peso corporal, foi calculada a eficiência alimentar, dividindo o ganho total de peso corporal (g) pela ingestão energética total (Kcal).

Foi avaliada a tolerância à glicose pelo teste oral de tolerância à glicose uma semana antes da eutanásia. Após um jejum de seis horas, foram coletadas amostras de sangue por punção da ponta da cauda na linha de base e após administração intraperitoneal de solução de glicose a $30 \%$ (Sigma-Aldrich ${ }^{\circledR}$, St Louis, MO, EUA), equivalente a $2,0 \mathrm{~g} / \mathrm{kg}$ de peso corporal. Foram analisadas as concentrações de glicose no sangue a 0 minutos (linha de base) e aos 15, 30, 60, 90 e 120 minutos de infusão de glicose, utilizando um glicosímetro portátil (Accu-chek Advantage; Roche Diagnostics Co., Indianápolis, IN, EUA). Foi avaliada a intolerância à glicose pela área sob a curva (AUC) para glicose.

Ao final do protocolo experimental, após jejum de 12 horas, os animais foram anestesiados (pentobarbital sódico $50 \mathrm{mg} / \mathrm{kg}$, injeção intraperitoneal), decapitados e toracotomizados; os diferentes depósitos de gordura do tecido adiposo foram dissecadas e pesadas. Foi calculada a GC como a soma do peso dos depósitos de gordura individuais da seguinte maneira: GC = gordura epididimal + gordura retroperitoneal + gordura visceral. Foi calculado o IA com a seguinte fórmula: IA = (GC/peso corporal final $)$ $\times 100$. As amostras de sangue foram coletadas em tubos heparinizados, centrifugados a $3.000 \times$ g por 10 minutos a $4^{\circ} \mathrm{C}$ e armazenados a $-80^{\circ} \mathrm{C}$ para análise subsequente. Foram determinados triacilglicerol, colesterol total, concentrações de lipoproteína de alta (HDL) e baixa densidade (LDL) utilizando kits específicos (BIOCLIN ${ }^{\circledR}$, Belo Horizonte, MG, Brasil). Os níveis hormonais de leptina e insulina foram determinados por ensaio de imunoabsorção enzimática (ELISA), utilizando kits comercialmente disponíveis (EMD Millipore Corporation, Billerica, MA, EUA).

Foi usado o modelo de avaliação da homeostase da resistência à insulina (HOMA-IR) como um índice de resistência à insulina, calculado de acordo com a fórmula: $\mathrm{HOMA}-\mathrm{IR}=$ [glicose em jejum $(\mathrm{mmol} / \mathrm{L}) \times$ insulina em jejum $(\mu \mathrm{U} / \mathrm{mL})] / 22,5.24$

\section{Perfil cardiovascular}

Foi avaliado também o perfil cardiovascular dos animais de acordo com os seguintes parâmetros: pressão arterial sistólica (PAS); morfologia do tecido cardíaco; expressão proteica miocárdica de colágeno tipo I, TIMP-1, TIMP-2 e leptina; e atividade da MMP-2.

\section{Pressão arterial sistólica}

Ao final do experimento, uma semana antes da eutanásia, foi medida a PAS em ratos conscientes utilizando o método não invasivo de manguito de cauda com um eletroesfigmomanômetro, Narco BioSystems ${ }^{\circledR}$ (International Biomedical, Austin, TX, EUA). ${ }^{25}$ As pulsações arteriais foram registradas em um sistema computadorizado de aquisição de dados (Biopac Systems Inc., CA, EUA). Foi registrada a média de duas leituras para cada medida.

\section{Estudo morfológico}

Os corações foram removidos e dissecados após a eutanásia e a toracotomia. O peso dos átrios e do ventrículos esquerdo e direito e as suas respectivas relações com o peso corporal final foram determinados com a finalidade de avaliar a presença de remodelação cardíaca (i.e., a presença ou ausência de hipertrofia).

\section{Níveis proteicos miocárdicos de colágeno tipo I, TIMP-1, TIMP-2 e leptina}

O tecido do ventrículo esquerdo foi analisado por western blot para quantificar os níveis proteicos de colágeno tipo I, TIMP-1, TIMP-2 e leptina. Foram utilizadas seis amostras em cada grupo para garantir que todas as amostras fossem analisadas na mesma corrida de eletroforese com a finalidade de evitar variações entre géis. Resumidamente, amostras congeladas do ventrículo esquerdo foram homogeneizadas usando um dispositivo Polytron (Ika Ultra TurraxTM T25 Basic, Wilmington, EUA) em tampão de lise contendo $10 \mathrm{mM}$ de Tris $\mathrm{pH} \mathrm{7,4,100} \mathrm{Mm} \mathrm{de} \mathrm{NaCl,} 1$ mM de EDTA, 1 Mm de EGTA, 1\% Triton X-100, 10\% glicerol, 0,1\% dodecil de sódio sulfato de sódio (SDS), desoxicolato a $0,5 \%$ e inibidores da fosfatase e protease (Sigma-Aldrich). O homogenato foi centrifugado a $4^{\circ} \mathrm{O}$ C durante 20 minutos a $12.000 \mathrm{rpm}$. Foi coletado o sobrenadante e o conteúdo total de proteínas foi determinado pelo método de Bradford. As amostras $(50 \mu \mathrm{g})$ foram submetidas a eletroforese em gel de SDSpoliacrilamida (SDS-PAGE) em géis de poliacrilamida $(6 \%$ ou 10\%, dependendo do peso molecular da proteína). Após eletroforese, as proteínas foram eletro-transferidas para uma membrana de nitrocelulose (BioRad Biosciences; NJ, EUA). A membrana foi subsequentemente bloqueada (5\% leite em pó desnatado, $10 \mathrm{mmol} / \mathrm{L}$ de Tris- $\mathrm{HCl} \mathrm{pH}$ 7,6, $150 \mathrm{mmol} / \mathrm{L}$ de $\mathrm{NaCl}$, e $0,1 \%$ Tween 20) durante 2 horas à temperatura ambiente e incubada com anticorpos específicos durante a

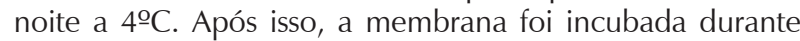
1,5 horas à temperatura ambiente com anticorpo secundário anti-coelho ou anti-camundongo conjugado com peroxidase (diluição 1:10.000) e subsequentemente incubada com quimioluminescência aumentada (Amersham Biosciences, NJ, USA) e detectado por autoradiografia. Foi realizada a análise de quantificação dos blots utilizando Scion Image software (Scion, baseado em NIH Image). Foram obtidos anticorpos monoclonais de camundongo para colágeno tipo I (1:10.000), TIMP-2 (1:1.000) e leptina $(1: 1.000)$ e anticorpos monoclonais de coelho para TIMP-1 (1:1.000) e $\beta$-actina $(1: 1.000)$ de Abcam (Cambridge, USA) e Cell Signaling (Danvers, USA), respectivamente. As bandas alvo foram normalizadas para a expressão da $\beta$-actina cardíaca. 


\section{Atividade da metaloproteinase-2 miocárdica}

A atividade da MMP-2 miocárdica foi determinada conforme descrito por Tyagi et al. ${ }^{26}$ Foram usadas seis amostras em cada grupo para garantir que todas as amostras fossem analisadas na mesma corrida de eletroforese para evitar variações entre géis. Resumidamente, os tecidos do ventrículo esquerdo foram homogeneizados em um tampão contendo: Tris $50 \mathrm{mM}, \mathrm{pH}$ 7,4, $\mathrm{NaCl}$ 0,2 M, Triton-X 0,1\% e CaCl 10 mM. O homogenato foi centrifugado a $4^{\circ} \mathrm{C}$ durante 20 minutos a $12.000 \mathrm{rpm}$. O sobrenadante foi coletado, e o conteúdo total de proteínas foi determinado pelo método de Bradford (Bradford 1976). As amostras foram diluídas em um tampão de aplicação contendo: 0,5 M Tris, pH 6,8, 100\% glicerol e 0.05\% azul de bromofenol. As amostras foram carregadas em poços de SDS-poliacrilamida a $8 \%$ contendo gelatina a $1 \%$. Foi realizada a eletroforese em um dispositivo Bio-Rad a $80 \mathrm{~V}$ durante duas horas. O gel foi removido e lavado duas vezes com Triton-X-100 a 2,5\% e subsequentemente lavado com $50 \mathrm{mM}$ de Tris, $\mathrm{pH}$ 8,4. O gel foi subsequentemente incubado a $37^{\circ} \mathrm{C}$ durante a noite em uma solução de ativação contendo $50 \mathrm{mM}$ de Tris, pH 8,4, $5 \mathrm{mM}$ de $\mathrm{CaCl} 2$ e $\mathrm{ZnCl} 2$. A coloração foi realizada durante 2 horas com azul de Coomassie a 0,5\%, e a descoloração foi realizada com metanol a 30\% e ácido acético a 10\% até que bandas claras fossem observadas sobre um fundo escuro. Os géis foram fotografados, e a intensidade da ação gelatinolítica (bandas claras) foi analisada em UVP, UV e um analisador de imagem White Darkhon.

\section{Análise estatística}

Antes da análise estatística, todos os dados foram testados quanto à normalidade usando o teste Shapiro-Wilk. Os resultados foram expressos como média \pm desvio padrão e submetidos ao teste t de Student para amostras independentes. O teste de correlação de Pearson foi utilizado para avaliar a associação entre as variáveis colágeno I, MMP-2, TIMP e leptina. Todas as análises estatísticas foram realizadas usando SigmaStat para Windows (Versão 3,5). O nível de significância considerado foi de $5 \%(\alpha=0,05)$.

\section{Resultados}

\section{Características gerais dos animais}

As características gerais dos animais encontram-se na Tabela 1. O peso corporal final; os depósitos de gordura epididimal, retroperitoneal e visceral; a GC total; e o IA foram significativamente mais altos no grupo obeso do que no grupo controle. Durante o período experimental, os animais no grupo obeso consumiram menos comida e calorias do que os animais do grupo controle; no entanto, a eficiência alimentar foi maior nos animais obesos.

\section{Perfis metabólicos e endócrinos}

Os perfis metabólicos e endócrinos estão resumidos na Figura 1. A obesidade a longo prazo induzida por alto teor de gordura levou a alterações metabólicas e hormonais significativas. Houve um aumento significativo na AUC de glicose, bem como nos níveis de insulina e leptina no grupo obeso, em comparação com o controle. Os animais obesos apresentaram um aumento da AUC, insulina sérica e HOMAIR. As medidas séricas de glicose, triacilglicerol, colesterol total, HDL e LDL foram semelhantes entre os grupos.

Tabela 1 - Características gerais dos animais

\begin{tabular}{|c|c|c|c|}
\hline \multirow{2}{*}{ Variáveis } & \multicolumn{3}{|c|}{ Grupos } \\
\hline & Controle $(n=20)$ & Obeso $(n=21)$ & Valor de $p$ \\
\hline $\mathrm{PCl}(\mathrm{g})$ & $151 \pm 11$ & $151 \pm 11$ & 0,290 \\
\hline$P C F(g)$ & $480 \pm 51$ & $534 \pm 58$ & 0,009 \\
\hline Gordura epididimal (g) & $9,3 \pm 2,3$ & $14,2 \pm 3,4$ & $<0,001$ \\
\hline Gordura epididimal/100g PCF & $1,9 \pm 0,5$ & $2,7 \pm 0,6$ & $<0,001$ \\
\hline Gordura retroperitoneal $(\mathrm{g})$ & $10,5 \pm 3,3$ & $21,7 \pm 5,9$ & $<0,001$ \\
\hline Gordura retroperitoneal $/ 100 \mathrm{~g}$ PCF & $2,2 \pm 0,7$ & $4,1 \pm 1,1$ & $<0,001$ \\
\hline Gordura visceral (g) & $6,3 \pm 1,4$ & $11,2 \pm 4,2$ & $<0,001$ \\
\hline Gordura visceral/100g PCF & $1,3 \pm 0,3$ & $2,1 \pm 0,8$ & $<0,001$ \\
\hline$G C(g)$ & $26,1 \pm 6,2$ & $47,2 \pm 12,3$ & $<0,001$ \\
\hline Índice de adiposidade & $5,6 \pm 0,9$ & $8,8 \pm 1,6$ & $<0,001$ \\
\hline Consumo alimentar (g/dia) & $22,8 \pm 2,1$ & $17,0 \pm 2,3$ & $<0,001$ \\
\hline Ingestão calórica (kcal) & $67,4 \pm 6,3$ & $62,1 \pm 8,2$ & 0,03 \\
\hline Eficiência alimentar (\%) & $2,1 \pm 0,2$ & $2,7 \pm 0,2$ & $<0,001$ \\
\hline
\end{tabular}

Valores são média \pm desvio padrão. GC: gordura corporal; PCF: peso corporal final; PCl: peso corporal inicial. Teste t de Student. 
A

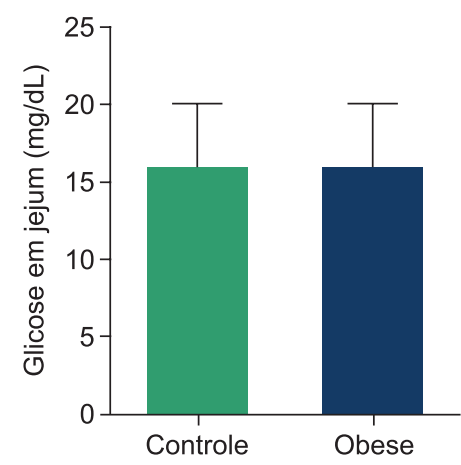

D

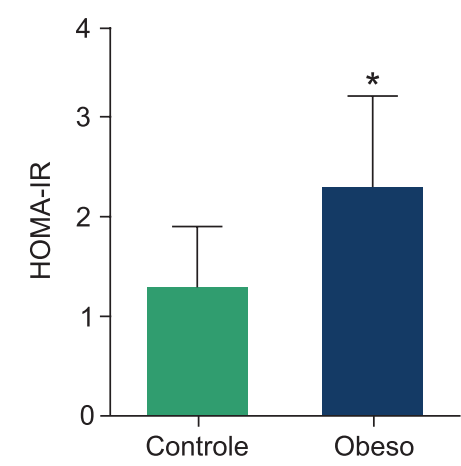

G

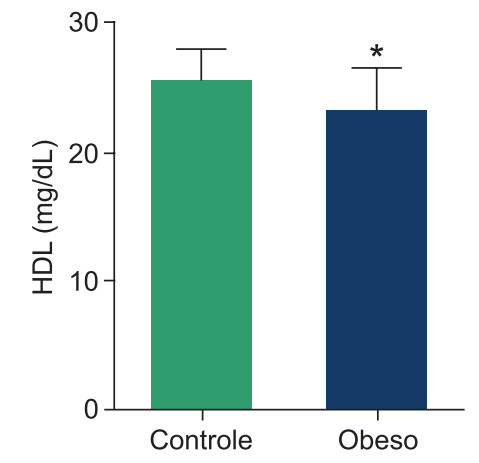

B

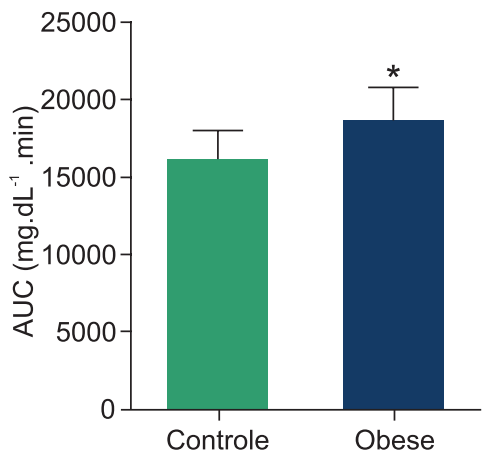

E

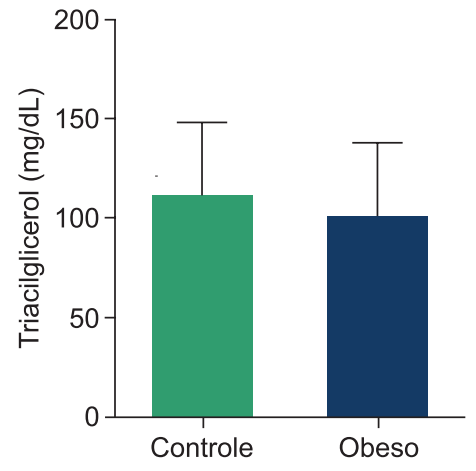

H

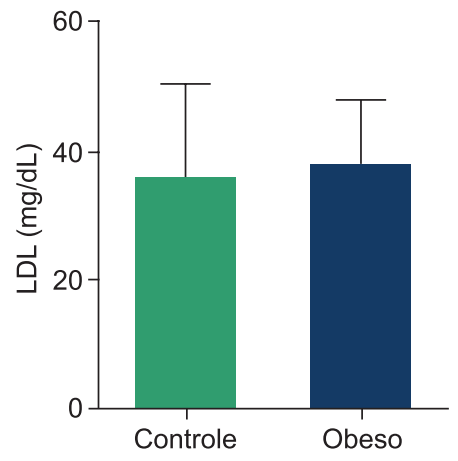

C

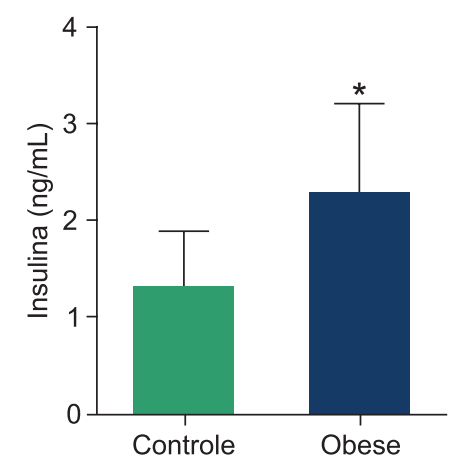

$\mathbf{F}$

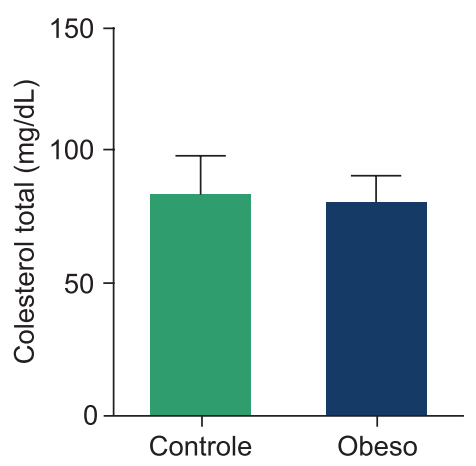

I

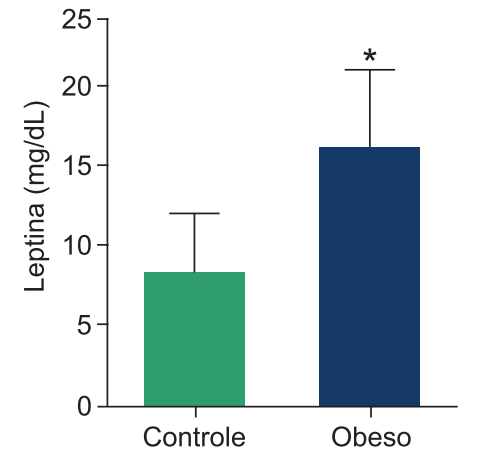

Figura 1 - Perfil metabólico e endócrino. (A) Glicose em jejum, (B) Área sob a curva (AUC) do teste intraperitoneal de tolerância à glicose, (C) insulina, (D) modelo de avaliação da homeostase da resistência à insulina (HOMA-IR), (E) triacilglicerol, (F) colesterol total, (G) lipoproteina de alta densidade (HDL), (H) lipoproteina de baixa densidade (LDL) e (I) leptina em ratos controles e obesos. ( $n=15-21$ por grupo). Dados são apresentados como média \pm desvio padrão; teste $t$ de Student. *: $p<0,05$.

Pressão arterial sistólica e perfil morfológico cardíaco

A Tabela 2 demonstra que a PAS foi mais alta nos animais obesos, e não houve diferenças significativas entre os grupos para quaisquer parâmetros estudados em relação ao perfil morfológico, exceto no ventrículo direito, sugerindo que a obesidade não desencadeou hipertrofia do ventrículo esquerdo.
Níveis proteicos miocárdicos de colágeno tipo I, TIMP-1, TIMP-2 e leptina

A Figura 2 demonstra que a obesidade promoveu uma redução dos níveis proteicos do colágeno tipo I, TIMP1 e TIMP-2; no entanto, houve um aumento dos níveis proteicos de leptina no grupo obeso em comparação ao grupo controle. 


\section{Artigo Original}

\section{Tabela 2 - Pressão arterial sistólica e perfil morfológico cardíaco}

\begin{tabular}{|c|c|c|c|}
\hline \multirow{2}{*}{ Variáveis } & \multicolumn{3}{|c|}{ Grupos } \\
\hline & Controle $(n=20)$ & Obeso $(n=21)$ & Valor de $p$ \\
\hline PAS & $127 \pm 11,0$ & $134 \pm 12,0$ & 0,04 \\
\hline Coração (g) & $1,10 \pm 0,10$ & $1,17 \pm 0,13$ & 0,06 \\
\hline $\mathrm{PA}(\mathrm{g})$ & $0,093 \pm 0,018$ & $0,094 \pm 0,021$ & 0,80 \\
\hline PVE (g) & $0,81 \pm 0,09$ & $0,82 \pm 0,10$ & 0,62 \\
\hline PVD (g) & $0,22 \pm 0,03$ & $0,24 \pm 0,03$ & 0,04 \\
\hline PA/PCF. $10^{-3}$ & $0,20 \pm 0,03$ & $0,18 \pm 0,03$ & 0,14 \\
\hline PVE /PCF. $10^{-3}$ & $1,72 \pm 0,11$ & $1,71 \pm 0,12$ & 0,44 \\
\hline PVD/PCF. $10^{-3}$ & $0,48 \pm 0,09$ & $0,47 \pm 0,05$ & 0,64 \\
\hline
\end{tabular}

Valores são médias \pm desvio padrão. PA: peso atrial; PAS: pressão arterial sistólica; PCF: peso corporal final; PVD: peso do ventrículo direito; PVE: peso do ventrículo esquerdo; relações PA/PCF; PVE/PCF; PVD/PCF; $10^{-3}=0,001$. Teste $t$ de Student.

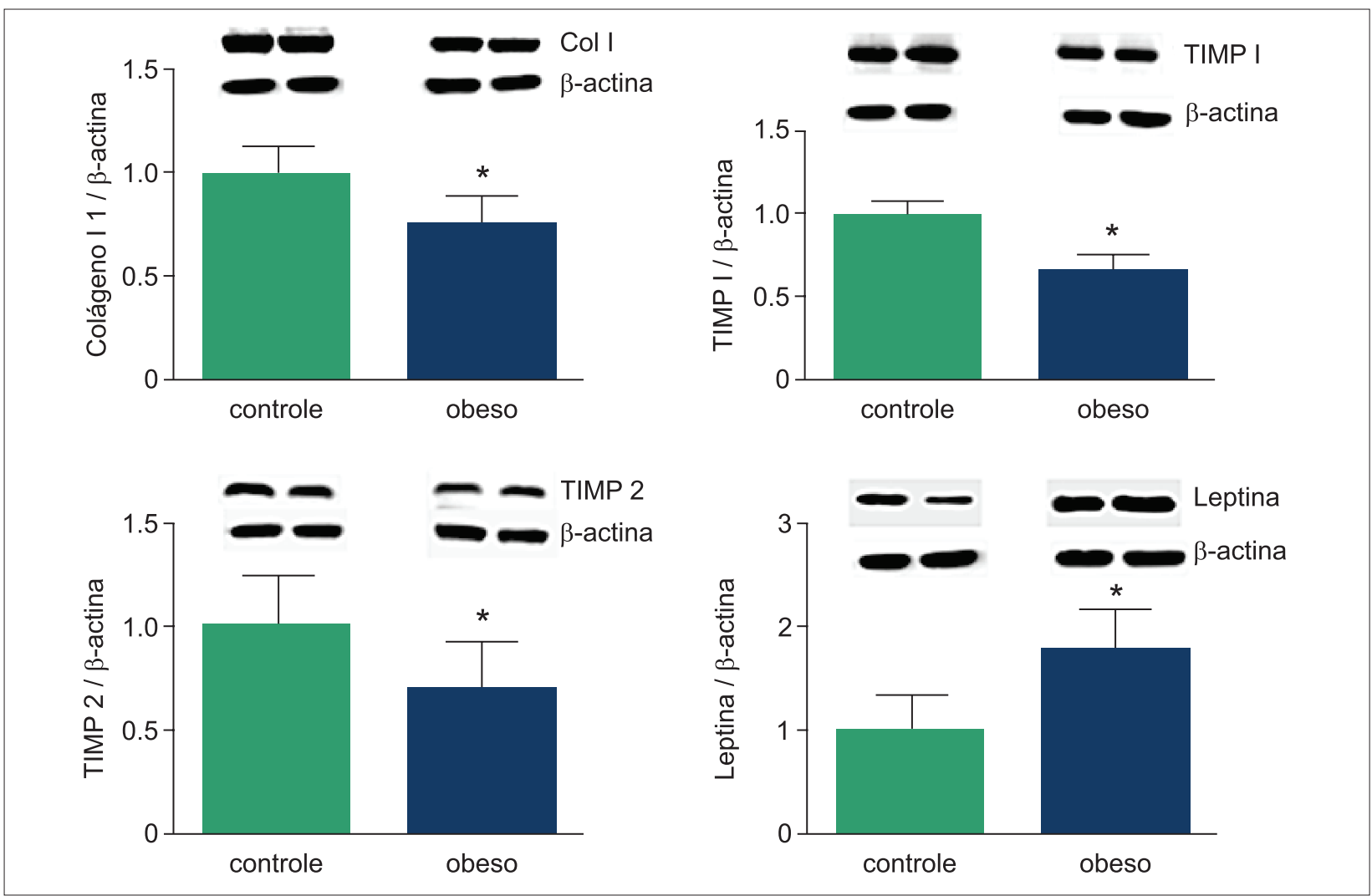

Figura 2 - Western blots representativos e quantificação de colágeno miocárdico tipo I, TIMP-1, TIMP-2 e leptina de ratos controles e obesos ( $n=6$ por grupo). As bandas dos western blot foram normalizados para $\beta$-actina. Dados são apresentados como média \pm desvio padrão; teste $t$ de Student. *: $p<0,05$.

\section{Atividade de MMP-2 miocárdica}

A Figura 3 mostra a identificação de duas bandas fracas de degradação correspondentes à MMP-2 no gel de eletroforese: a MMP-2 inativa (pró-MMP-2) com um peso molecular de aproximadamente 72 kDa e MMP-2 ativa com um peso molecular de aproximadamente $64 \mathrm{kDa}$. Entre as duas bandas mencionadas, foi possível identificar a banda forte da degradação intermediária da MMP-2. Houve um aumento significativo na MMP-2 nos animais obesos.

\section{Associação linear entre variáveis cardíacas}

A Tabela 3 demonstra que houve uma correlação significativa entre a redução do colágeno tipo I e o aumento da atividade da MMP-2, bem como entre a elevação da 


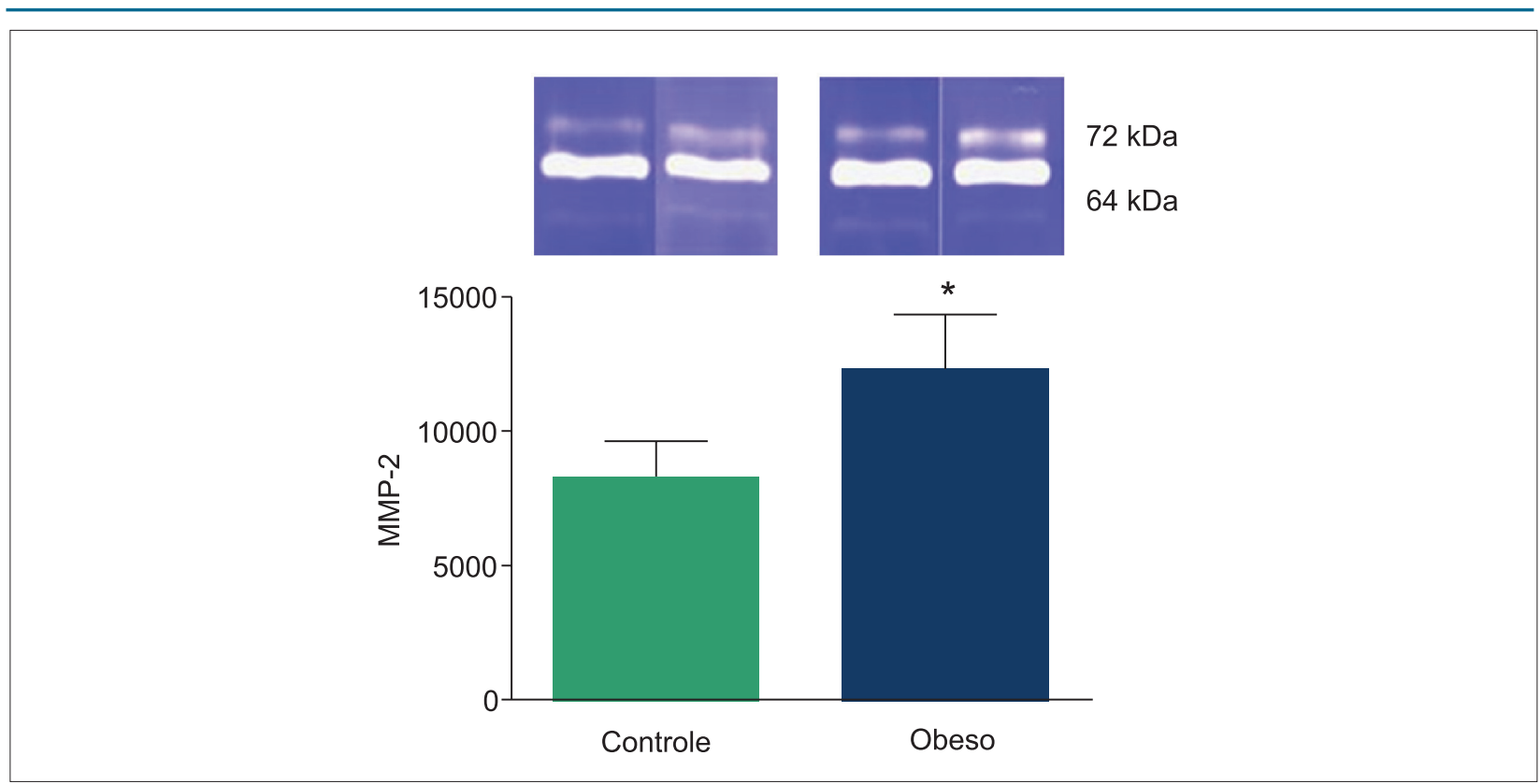

Figura 3 - Relação entre MMP-2 ativa (ativa e ativa intermediária) e inativa em ratos controles e obesos ( $n=6$ por grupo). MMP-2 inativa = $72 \mathrm{kDa}$ e MMP-2 ativa = 64 $k D a$. Dados são apresentados como média \pm desvio padrão; teste $t$ de Student. *: $p<0,05$.

atividade da MMP-2 e a leptina. Além disso, foi observada uma correlação entre o aumento da MMP-2 e a redução do TIMP-1 e -2, bem como entre a redução do TIMP-1 e o aumento dos níveis proteicos de leptina.

\section{Discussão}

O presente estudo visou investigar se a redução do colágeno tipo I está associada ao aumento da atividade da MMP-2 e a níveis elevados de leptina no miocárdio de ratos obesos. Os resultados principais confirmaram esta hipótese.

A alimentação contínua com uma dieta com alto teor de gordura foi eficaz para promover a obesidade em 34 semanas, uma vez que os ratos apresentaram níveis mais elevados de peso corporal, gordura e IA em comparação aos ratos alimentados com uma dieta padrão; tais dados corroboram outros estudos. ${ }^{27,28}$

As principais causas da obesidade são: o suprimento mais abundante de alimentos, a maior ingestão de alimentos palatáveis e densos em energia e a redução do gasto energético. A dieta com alto teor de gordura utilizada no presente estudo era rica em ácidos graxos mono e poliinsaturados com um conteúdo energético de $3,65 \mathrm{kcal} / \mathrm{g}$, enquanto a dieta padrão dada ao grupo controle consistia em 2,95 kcal/g, gerando uma diferença de $24 \%$ em conteúdo calórico. Outros autores têm demonstrado que o consumo de uma dieta com alto teor de gordura promove menos saciedade e assim aumenta a ingestão de alimentos. ${ }^{29}$

Esses dados diferem dos nossos resultados, visto que os animais obesos ingeriram uma quantidade menor de alimentos e calorias em comparação ao controle. No entanto, a eficiência alimentar era mais alta nos ratos obesos, provavelmente devido ao efeito térmico do alimento. A gordura alimentar
Tabela 3 - Associação linear entre variáveis cardíacas

\begin{tabular}{lcc}
\hline Associação & $\begin{array}{c}\text { Coeficiente de } \\
\text { correlação }\end{array}$ & Valor de $\mathbf{p}$ \\
\hline Colágeno I $\times$ MMP-2 & $-0,723$ & 0,008 \\
\hline MMP-2 $\times$ Leptina & 0,766 & 0,004 \\
\hline MMP-2 $\times$ TIMP-1 & $-0,815$ & 0,001 \\
\hline MMP-2 $\times$ TIMP-2 & $-0,597$ & 0,040 \\
\hline TIMP-1 $\times$ Leptina & $-0,656$ & 0,020 \\
\hline TIMP-2 $\times$ Leptina & $-0,273$ & 0,390 \\
\hline
\end{tabular}

Valores são médias \pm desvio padrão. PA: peso atrial; PAS: pressão arterial sistólica; PCF: peso corporal final; PVD: peso do ventrículo direito; PVE: peso do ventrículo esquerdo; relações PA/PCF; PVE/PCF; PVD/PCF; $10^{-3}=0,001$. Teste $t$ de Student.

requer menos energia ( $2 \%-3 \%)$ para ser metabolizada, e a gordura excessiva é, portanto, facilmente depositada na forma de triglicerídeos nos adipócitos, resultando em obesidade. ${ }^{30}$

Vários estudos têm relatado algumas comorbidades relacionadas à obesidade $29,31,32$ tais como intolerância à glicose, resistência à insulina, dislipidemia, hiperinsulinemia, hiperleptinemia e hipertensão arterial. No presente estudo, os animais obesos apresentaram maior AUC no teste oral de tolerância à glicose e maiores níveis séricos de insulina que os controles, indicando que a obesidade promoveu intolerância à glicose e hiperinsulinemia. A intolerância à glicose, associada ao aumento da insulina sérica, indicou que os ratos obesos apresentaram resistência à ação da insulina. Estes resultados são ainda corroborados pelo aumento do HOMA-IR nos ratos obesos. Todos estes achados estão de acordo com relatos prévios que utilizaram ratos alimentados com uma dieta com 
alto teor de gordura insaturada. ${ }^{13,27,28,33}$ Diversos estudos têm mostrado que a obesidade induzida por uma dieta com alto teor de gordura leva à dislipidemia, ${ }^{19,34,35}$ devido a mudanças em triacilglicerol, LDL ou HDL. Em nosso estudo, foi observada a redução dos níveis séricos de HDL. A leptina é um hormônio produzido pelo tecido adiposo, que participa do balanço energético, por meio da regulação da ingestão de alimentos e da oxidação de lipídios, ${ }^{36}$ e da biologia dos colágenos. ${ }^{5,14-16}$

Em relação aos efeitos da obesidade no sistema cardiovascular, a obesidade não promoveu remodelação cardíaca do ventrículo esquerdo. No entanto, a PAS aumentou nos animais obesos. Visto que o controle da PAS envolve o sistema neuro-humoral, como o sistema nervoso simpático e o sistema renina-angiotensina-aldosterona, os quais são aumentados na obesidade, ${ }^{37}$ pode-se inferir que o sistema neuro-humoral é ativado em animais obesos. Este achado está em acordo com alguns pesquisadores anteriores que investigaram a PAS em animais obesos alimentados com uma dieta com alto teor de gordura ${ }^{38}$ e em desacordo com outros. ${ }^{27}$

O objetivo principal deste estudo foi investigar se o aumento da atividade da MMP-2 pela leptina é responsável pela redução do colágeno miocárdico tipo I em ratos obesos. Os resultados desta investigação indicaram que houve uma redução dos níveis proteicos do colágeno tipo I acompanhada por um aumento da atividade da MMP-2 e dos níveis proteicos da leptina e uma redução dos níveis proteicos de TIMP-1 e TIMP-2 no coração. Como previamente referido, poucos estudos têm avaliado o comportamento do colágeno tipo I no miocárdio de animais com obesidade induzida por uma dieta com alto teor de gordura; enquanto Carroll e Tyaggi ${ }^{12} \mathrm{e}$ Martínez-Martínez ${ }^{39}$ verificaram um aumento, Silva et al., ${ }^{13}$ verificou uma redução do colágeno miocárdico tipo I.

As alterações no colágeno miocárdico podem resultar de uma elevação da síntese ou uma diminuição da degradação. Os dados deste estudo demonstraram que a degradação do colágeno tipo I pode ter prevalecido em ratos obesos, considerando que houve uma associação significativa entre o colágeno tipo I reduzido e o aumento da atividade da MMP-2. Embora alguns estudos indiquem que o aumento da atividade da MMP-2 melhora a síntese de colágeno, ${ }^{40}$ a maioria das informações na literatura indica o comportamento oposto, i.e., o aumento da atividade da MMP-2 promove a degradação do colágeno tipo I. 5,39,41 Embora Martínez-Martínez et al., ${ }^{39}$ e Zibadi et al., ${ }^{14}$ tenham verificado que a leptina reduziu a atividade da MMP-2 in vitro, outros estudos têm demonstrado que a leptina aumenta a atividade da MMP-2 ${ }^{5,15,16}$ e os nossos resultados corroboram ainda mais este último achado. Portanto, a elevação da MMP-2 pode ter sido consequente ao aumento da leptina cardíaca, porque houve uma estreita associação entre estas duas variáveis, embora esses achados não necessariamente reflitam uma relação de causa e efeito. Apesar disso, vários estudos têm relatado uma relação direta entre a leptina e a atividade da MMP-2 em cardiomiócitos. 5,15,16

Apesar do fato que o aumento da atividade da MMP está associado à elevação da leptina cardíaca, outro fator modificante dessa enzima é o comportamento dos TIMP. Os resultados do presente estudo demonstraram uma redução dos níveis proteicos de TIMP-1 e TIMP-2 em animais obesos, que pode ter influenciado o aumento da MMP-2, uma vez que houve uma associação significativa entre MMP-2 e TIMP-1 e TIMP-2. A redução do TIMP-1 pode estar relacionada ao aumento da leptina, uma vez que houve uma associação significativa entre estas variáveis. Este achado é consistente com Schram et al., que verificaram uma redução substancial na expressão do mRNA de TIMP-1 após a elevação das concentrações da leptina em células cardíacas cultivadas. ${ }^{15}$ Até onde sabemos, este é o primeiro estudo que avalia a associação entre colágeno tipo I, leptina, MMP-2 e TIMP-1 e TIMP-2 no miocárdio de animais obesos alimentados com uma dieta com alto teor de gordura insaturada. No entanto, outras análises são necessárias para confirmar a relação de causa e efeito.

\section{Conclusão}

Os achados confirmaram a hipótese de que a redução do colágeno tipo I está associada ao aumento da atividade da MMP-2, que está, por sua vez, ligada à elevação da leptina no miocárdio de ratos obesos. Este estudo permitiu a avaliação de mediadores envolvidos na remodelação cardíaca, os quais podem desencadear a função cardíaca comprometida na obesidade. A identificação desses fatores deletérios pode facilitar possíveis alvos terapêuticos.

\section{Contribuição dos autores}

Concepção e desenho da pesquisa: Silva-Bertani DCT, Padovani CR, Cicogna AC; Obtenção de dados: Silva-Bertani DCT, Vileigas DF, De Tomasi LC, Campos DHS, Deus AF, Freire PP, Alves CAB; Análise e interpretação dos dados: SilvaBertani DCT, Vileigas DF, Mota GAF, Souza SLB, De Tomasi LC, Campos DHS, Deus AF, Freire PP, Alves CAB, Cicogna AC; Análise estatística: Silva-Bertani DCT, Padovani CR, Cicogna AC; Obtenção de financiamento: Silva-Bertani DCT, Cicogna AC; Redação do manuscrito: Silva-Bertani DCT, Vileigas DF, Mota GAF, Souza SLB, Cicogna AC; Revisão crítica do manuscrito quanto ao conteúdo intelectual importante: SilvaBertani DCT, Vileigas DF, Mota GAF, Souza SLB, Cicogna AC.

\section{Potencial conflito de interesses}

Declaro não haver conflito de interesses pertinentes.

\section{Fontes de financiamento}

O presente estudo foi financiado pela FAPESP (2010/142084 e 2011/12165-9).

\section{Vinculação acadêmica}

Este artigo é parte de dissertação de Mestrado de Danielle Cristina Tomaz da Silva-Bertani pela Faculdade de Medicina de Botucatu (UNESP).

\section{Aprovação ética e consentimento informado}

Este estudo foi aprovado pelo Comitê de Ética da Faculdade de Medicina de Botucatu sob o número de protocolo 861-2011. Todos os procedimentos envolvidos nesse estudo estão de acordo com a Declaração de Helsinki de 1975, atualizada em 2013. 


\section{Referências}

1. World Health Organization.(WHO). Fact sheets: Obesity and overweight (Internet). (Cited 2018 Jan 18). Available from: https://www.who.int/newsroom/fact-sheets/detail/obesity-and-overweight.

2. Bhurosy T, Jeewon R. Overweight and obesity epidemic in developing countries: a problem with diet, physical activity, or socioeconomic status? Scient World J. 2014;2014:964236.

3. Hall ME, Harmancey R, Stec DE. Lean heart: Role of leptin in cardiac hypertrophy and metabolism. World J Cardiol. 2015;7(9):511-24.

4. Segura AM, Frazier OH, Buja LM. Fibrosis and heart failure. Heart Fail Rev. 2014;19(2):173-85.

5. Berg G, Schreier L, Miksztowicz V. Circulating and adipose tissue matrix metalloproteinases in cardiometabolic risk environments: pathophysiological aspects. Horm Mol Biol Clin Investig. 2014;17(2):79-87.

6. Matsubara LS, Narikawa S, Ferreira ALA, Paiva SA, Zornoff LM et al. Remodelação miocárdica na sobrecarga crônica de pressão e volume no coração de ratos. Arq Bras Cardiol. 2006;86(2):126-30.

7. Alberts B, Johnson A, Lewis J, Raff M, Roberts $K$ et al. Junções celulares, adesão celular e matriz extracelular. In: Alberts B. Biologia molecular da célula. 5th ed. Porto Alegre: Artmed; 2010. 1131-204 p.

8. Berk BC, Fujiwara K, Lehoux S. MEC remodeling in hypertensive heart disease. J Clin Invest. 2007;117(3):568-75.

9. Szcz $\square$ sny W, Szczepanek J, Tretyn A, D $\square$ browiecki S, Szmytkowski J, Polak J. An analysis of the expression of collagen I and III genes in the fascia of obese patients. J Surg Res. 2015 May;195(2):475-80.

10. Lancha A, Rodríguez A, Catalán V, Becerril S, Sáinz N, Ramírez B, et al. Osteopontin deletion prevents the development of obesity and hepatic steatosis via impaired adipose tissue matrix remodeling and reduced inflammation and fibrosis in adipose tissue and liver in mice. PLoS One. 2014;9(5):e98398.

11. Minullina IR, Alexeyeva NP, Anisimov S V, Puzanov MV, Kozlova SN, Sviryaev $\mathrm{Y} V$, et al. Transcriptional changes in bone marrow stromal cells of patients with heart failure. Cell Cycle. 2014;13(9):1495-500.

12. Carroll JF, Tyagi SC. Extracellular matrix remodeling in the heart of the homocysteinemic obese rabbit. Am J Hypertens. 2005;18(5 Pt 1):692-8.

13. Silva DCT da, Lima-Leopoldo AP, Leopoldo AS, Campos DHS de, Nascimento AF do, Oliveira Junior SA de, et al. Influence of term of exposure to high-fat diet-induced obesity on colágeno miocárdico tipo I and III. Arq Bras Cardiol. 2014;102(2):157-63.

14. Zibadi S, Cordova F, Slack EH, Watson RR, Larson DF. Leptin's regulation of obesity-induced cardiac extracellular matrix remodeling. Cardiovasc Toxicol. 2011;11(4):325-33.

15. Schram K, De Girolamo S, Madani S, Munoz D, Thong F, Sweeney G. Leptin regulates MMP-2, TIMP-1 and collagen synthesis via p38 MAPK in HL-1 murine cardiomyocytes. Cell Mol Biol Lett. 2010;15(4):551-63.

16. Schram K, Ganguly R, No EK, Fang X, Thong FSL, Sweeney G. Regulation of MT1-MMP and MMP-2 by leptin in cardiac fibroblasts involves Rho/ ROCK-dependent actin cytoskeletal reorganization and leads to enhanced cell migration. Endocrinology. 2011;152(5):2037-47.

17. Deus AF, Vileigas DF, Silva DCT, Tomasi LC, Campos DHS, Okoshi K, et al. Cardiac function and intracellular $\mathrm{Ca} 2+$ handling proteins are not impaired by high-saturated-fat diet-induced obesity. Braz J Med Biol Res. 2019;52(6):e8085.

18. Jacobsen BB, Leopoldo APL, Cordeiro JP, Campos DHS de, Nascimento AF do, Sugizaki MM, et al. Cardiac, Metabolic and Molecular Profiles of Sedentary Rats in the Initial Moment of Obesity. Arq Bras Cardiol. 2017;109(5):432-9.
19. Vileigas DF, de Deus AF, da Silva DCT, de Tomasi LC, de Campos DHS, Adorni CS, et al. Saturated high-fat diet-induced obesity increases adenylate cyclase of myocardial $\square$-adrenergic system and does not compromise cardiac function. Physiol Rep. 2016;4(17):pii e12914.

20. National Institute of Health. Guide for the care and use of laboratoty animals. [Contract NOI-RR-2-2135]. Bethesda:Animal Resources Program. Division of Research Resources; 1985.

21. Rodríguez-Calvo R, Girona J, Alegret JM, Bosquet A, Ibarretxe D, Masana L. Role of the fatty acid-binding protein 4 in heart failure and cardiovascular disease. J Endocrinol. 2017;233(3):R173-84.

22. Zhang J, Qiao C, Chang L, Guo Y, Fan Y, Villacorta L, et al. Cardiomyocyte Overexpression of FABP4 Aggravates Pressure Overload-Induced Heart Hypertrophy. PLoS One. 2016;11(6):e0157372.

23. Aasum E, Khalid AM, Gudbrandsen OA, How O-J, Berge RK, Larsen TS Fenofibrate modulates cardiac and hepatic metabolism and increases ischemic tolerance in diet-induced obese mice. J Mol Cell Cardiol. 2008;44(1):201-9.

24. Matthews DR, Hosker JP, Rudenski AS, Naylor BA, Treacher DF, Turner RC. Homeostasis model assessment: insulin resistance and beta-cell function from fasting plasma glucose and insulin concentrations in man. Diabetologia. 1985;28(7):412-9.

25. Santos PP Dos, Rafacho BPM, Gonçalves A de F, Jaldin RG, Nascimento TB do, Silva MAB, et al. Vitamin D induces increased systolic arterial pressure via vascular reactivity and mechanical properties. PLoS One. 2014;9(6):e98895.

26. Tyagi SC, Matsubara L, Weber KT. Direct extraction and estimation of collagenase(s) activity by zymography in microquantities of rat myocardium and uterus. Clin Biochem. 1993;26(3):191-8.

27. Nascimento AF, Luvizotto RAM, Leopoldo AS, Lima-Leopoldo AP, Seiva FR, Justulin LA, et al. Long-term high-fat diet-induced obesity decreases the cardiac leptin receptor without apparent lipotoxicity. Life Sci. 2011;88(2324):1031-8.

28. Lima-Leopoldo AP, Leopoldo AS, Sugizaki MM, Bruno A, Nascimento AF Luvizotto RAM, et al. Disfunção miocárdica e alterações no trânsito de cálcio intracelular em ratos obesos. Arq Bras Cardiol. 2011;97(3):232-40.

29. Carroll JF, Zenebe WJ, Strange TB. Cardiovascular function in a rat model of diet-induced obesity. Hypertens (Dallas, Tex 1979). 2006;48(1):65-72.

30. Jéquier E. Pathways to obesity. Int J Obes Relat Metab Disord. 2002;26 Suppl 2:S12-7.

31. Nivoit P, Morens C, Van Assche FA, Jansen E, Poston L, Remacle C, et al. Established diet-induced obesity in female rats leads to offspring hyperphagia, adiposity and insulin resistance. Diabetologia. 2009;52(6):1133-42.

32. Relling DP, Esberg LB, Fang CX, Johnson WT, Murphy EJ, Carlson EC, et al. High-fat diet-induced juvenile obesity leads to cardiomyocyte dysfunction and upregulation of Foxo3a transcription factor independent of lipotoxicity and apoptosis. J Hypertens. 2006;24(3):549-61.

33. Omar B, Pacini G, Ahrén B. Differential development of glucose intolerance and pancreatic islet adaptation in multiple diet induced obesity models. Nutrients. 2012;4(10):1367-81.

34. Leopoldo AS, Lima-Leopoldo AP, Sugizaki MM, do Nascimento AF, de Campos DHS, Luvizotto R de AM, et al. Involvement of L-type calcium channel and SERCA2a in myocardial dysfunction induced by obesity. J Cell Physiol. 2011;226(11):2934-42.

35. Ferron AJT, Jacobsen BB, Sant'Ana PG, de Campos DHS, de Tomasi LC, Luvizotto R de AM, et al. Cardiac Dysfunction Induced by Obesity Is Not Related to $\beta$-Adrenergic System Impairment at the Receptor-Signalling Pathway. PLoS One. 2015;10(9):e0138605. 


\section{Artigo Original}

36. Yang R, Barouch LA. Leptin signaling and obesity: cardiovascular consequences. Circ Res. 2007;101(6):545-59.

37. Srinivasan SR, Bao W, Wattigney WA, Berenson GS. Adolescent overweight is associated with adult overweight and related multiple cardiovascular risk factors: the Bogalusa Heart Study. Metabolism. 1996;45(2):235-40.

38. Fitzgerald SM, Henegar JR, Brands MW, Henegar LK, Hall JE. Cardiovascular and renal responses to a high-fat diet in Osborne-Mendel rats. Am J Physiol Regul Integr Comp Physiol. 2001;281(2):R547-52.

39. Martínez-Martínez E, Jurado-López R, Valero-Muñoz M, Bartolomé MV, Ballesteros S, Luaces M, et al. Leptin induces cardiac fibrosis through galectin-3, mTOR and oxidative stress: potential role in obesity. J Hypertens. 2014;32(5):1104-14; discussion 1114.

40. Hori Y, Kashimoto T, Yonezawa T, Sano N, Saitoh R, Igarashi S, et al. Matrix metalloproteinase-2 stimulates collagen-I expression through phosphorylation of focal adhesion kinase in rat cardiac fibroblasts. Am J Physiol Cell Physiol. 2012;303(9):C947-53.

41. Laviades C, Varo N, Fernández J, Mayor G, Gil MJ, Monreal I, et al. Abnormalities of the extracellular degradation of colágeno tipo I in essential hypertension. Circulation. 1998;98(6):535-40. 